\title{
Taking Advantage of Co(II) Induced Enhanced VCD for the Fast and Sensitive Determination of Enantiomeric Excess
}

Lorenzo Arrico, ${ }^{a}$ Gaetano Angelicia and Lorenzo Di Bari*a

Co(II) induces large VCD signal enhancement, which overcomes the low sensitivity of VCD and opens new perspectives in quantitative VCD. We used a stereodynamic system, where the analyte determines the chirality of the Co(II) coordination sphere. The strong VCD signals stand out the baseline so well to allow for their rapid and accurate quantitation. This lends itself to the first general method for the e.e. determination of $\alpha$-amino acids based on vibrational circular dichroism and constitutes a model for the design of sensitive quantitative essays based on VCD.

The importance of enantiomeric excess (e.e.) determination in pharmaceutical, food and fragrances chemistry, as well as in asymmetric synthesis and catalysis, is widely known. Currently, there are several methodologies to accurately determine enantiomeric composition. Each of them, from NMR spectroscopy to chromatographic and chiroptical methods, presents advantages and drawbacks, in terms of costs and time, resulting in a constant need for innovative solutions. Moreover, it's worth to recall that, to reach today's efficiency, all of them required years of technological progress, since their first introduction. A current pressing challenge comes from the need to implement high throughput screening for optimizing asymmetric synthesis reaction conditions. To develop such technology, it's essential to have a fast method, suitable for automation, which can selectively operate on a range of different substrates, even in complex matrices.

Chiroptical methods are the natural tool to investigate chirality and they are particularly appealing for the determination of enantiomeric compositions, saving time and costs for solvents/chiral columns, compared to the more widespread chromatographic separations. Innumerable methods for e.e. determination, based on electronic circular dichroism (ECD) have been reported for different classes of molecules, e.g. carboxylic acids, ${ }^{1}$ alcohols, ${ }^{2}$ amines, ${ }^{3}$ diamines, ${ }^{4}$ amino alcohols, ${ }^{5}$ hydroxy carboxylic acids, ${ }^{6}$ and aldehydes. ${ }^{7}$ On the contrary, only few examples of vibrational circular dichroism (VCD) e.e. determination have been reported so far. ${ }^{8,9}$

In principle VCD measurements, alongside with Raman optical activity (ROA) ones, could cover a much larger scope of molecules. As a matter of fact, such vibrational optical activity (VOA) spectroscopies are not limited to molecules with a suitable UV-vis spectrum (i.e. containing chromophores) and moreover, additional structural information are provided along the IR/Raman spectra acquisition. Unfortunately, VOA suffer from weak intensities of the optical bands, so that long acquisition time and concentrated sample are required to obtain good signal (S) to noise (N) ratio. However, it was reported that the presence of metal ions can induce signal enhancement both in $\mathrm{ROA}^{10}$ and VCD spectroscopies. In the latter case, the observation of metal ions-induced VCD signals enhancement dates back to $1980,{ }^{11}$ but, despite some renewed interest, ${ }^{12-15}$ this effect has remained largely unexplored until lately. Although not completely clear, the most commonly

\footnotetext{
a Dipartimento di Chimica e Chimica Industriale, Università di Pisa, via G. Moruzzi 13, I-56124 Pisa, Italy. Email: lorenzo.dibari@unipi.it

Electronic Supplementary Information (ESI) available: experimental details (synthesis, spectral acquisition procedure and parameters), ECD spectra of Trp-Co, IR absorption and VCD spectra of Ala-Co, Phe-Co and Trp-Co.
} 
accepted explanation for such enhancement is to be found in the coupling between the magnetic dipole transition (MDT) of electronic transition, in the presence of low-lying electronic states (LLESs), and the vibrational ones. ${ }^{13,16}$ Unfortunately, current computational methods do not allow for the calculation of this enhancement.

We recently introduced a new concept in the field of VCD analysis: the use of the extraordinary magnitude of VCD signals found in Co(II) complexes for the chirality recognition of $\alpha$-amino acids. ${ }^{17}$ Herein, we present a step toward the exploitation of $\mathrm{Co}(\mathrm{II})$-induced giant signals to overcome the limits of analytical applications of VCD, usually affected not only by weak signal intensities but also by an instrumental baseline drift or distortion, which make quantitative VCD applications difficult to realize and finally less attractive. We decided to use chiral $\alpha$-amino acids as model molecules for our analysis, following our previous work. ${ }^{17}$ Our concept is simple, and it is largely inspired by successful protocols, developed for high throughput and sensitive analysis by means of ECD. ${ }^{1-3,7,18,19} \mathrm{~A}$ stereodynamic metal complex is used as a reporter of chirality of the target analytes. We took inspiration from a recently developed self-assembling system where the chirality of the amino acid is incorporated into a Co(II) complex (probe). ${ }^{17,20,21}$ The probe presents an achiral ligand, which is a tris(pyridylmethyl)amine (TPA) derivative. In the absence of chiral elements, TPA-based complexes exist as a dynamic mixture of two enantiomeric propeller-like conformations, the clockwise $(P)$ and counter-clockwise $(\mathrm{M})$ forms. When a chiral perturbation is introduced, either with a stereogenic centre on the backbone of TPA or with the addition of a chiral ancillary ligand, one enantiomeric conformation is favoured upon the other one., ${ }^{1,22-24}$ In our case, chirality becomes embedded in the $\mathrm{Co}(\mathrm{II})$ complex by the formation of a dynamic covalent adduct between the probe and the chiral analyte, via a reversible imine and the coordination of the carboxylic acid to the metal centre (Scheme 1).

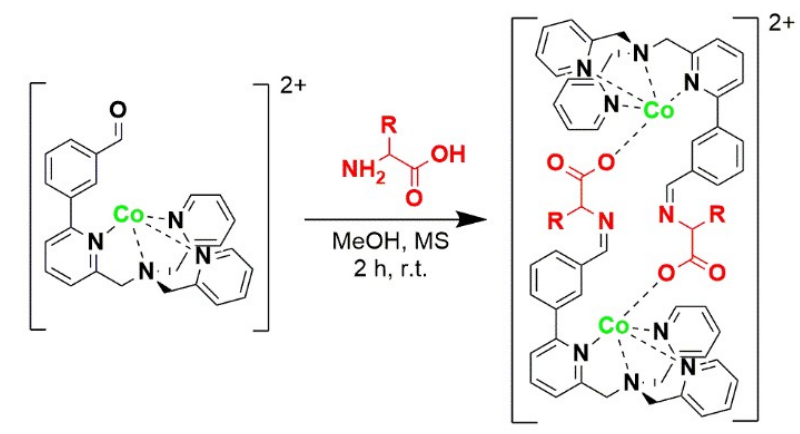

Scheme 1: Synthetic scheme for the self-assembly of the Aaa-Co complexes. Perchlorate is the counterion.

The chiral probe-analyte complex (Aaa-Co) is depicted as binuclear, in analogy to the previously described $\mathrm{Zn}$ (II) complexes, ${ }^{21}$ even though we have not yet gained direct evidence of the structure in the present case. The use of $\mathrm{Zn}(\mathrm{II})$-based probe was reported for the determination of the enantiomeric excess of amino acids by means of ECD. Very recently, we used related trinuclear $\mathrm{Co}$ (II) systems for a simple, sensitive and rapid recognition of the absolute configuration (AC) of amino acids via $\mathrm{VCD} .{ }^{17}$ Thanks to the giant VCD signals, we were able to record quantitative and conclusive spectra in few minutes and with small amounts of analyte. Moreover, independently of the nature and bulkiness of the aminoacid sidechains, we measured VCD spectra with the same signals patterns, thus obtaining a fingerprint of the AC. This interesting feature essentially arises from the distancerelated character of the VCD enhancement: as demonstrated by Buma, Woutersen and coworkers, 
only the oscillators which are in close proximity to the Co(II) ion can undergo the amplification of their VCD bands. ${ }^{14,16}$ The chiral analyte dictates the helicity of the Co(II)-TPA core, whose vibrational modes are near to the focus of enhancement, while all other oscillators allied for example on the aminoacid itself are too far away to gain extra rotator strength. A further aspect, which at the moment lacks theoretical support, is the geometry, or better the symmetry of the normal modes undergoing enhancement: in order to give rise to an effective coupling to the magnetic dipole, they must belong to the same irreducible representation, otherwise the interaction will be vanishing.

After demonstrating this qualitative application, useful for AC recognition, we turned onto quantitative analysis, i.e. the determination of the enantiomeric composition. In this case, we took advantage of: 1 ) the very high $\mathrm{S} / \mathrm{N}$ of the giant signals; 2 ) the fact that the baseline problems, which plague VCD measurements, become irrelevant.

Before describing in detail our results, a clarification is in order. Zonta and co-workers proposed two variations of an ECD-active Zn(II) complex. Initially, they suggested a system based on a 1:1 stoichiometry between the chiral $\alpha$-amino acid and the $\mathrm{Zn}$ (II)-(TPA derivative) probe. In their original paper, ${ }^{20}$ they hypothesized a mononuclear complex, which thereafter appeared as a $C_{2}$-symmetric dinuclear dimer. More recently, they found that by adding $\mathrm{Zn}(\mathrm{II})$ in excess, they obtained a trinuclear dimer with somewhat stronger ECD features. ${ }^{21}$ In our previous investigation of Co(II)-induced VCD enhancement, we used the trinuclear complex, whose assembly requires 12 hours of reaction time. Looking for the best compromise between ease of preparation and signal enhancement, we investigated the pristine dinuclear system, which is easily obtained by mixing Co(II) probe and amino acid in 1:1 stoichiometry. $¥$ In analogy to the ECD behaviour, the VCD signals of the dinuclear systems are less intense compared to those of the trinuclear ones, i.e. the dissymmetry factors of our Aaa-Co are 2-3 times lower than those of the trinuclear dimers. Nonetheless, we chose to use the dinuclear Aaa-Co complexes to avoid the drawbacks of the trinuclear systems, namely the longer reaction time and the more complex equilibrium mixture. The latter one is particularly detrimental when no purification is carried out, because in the assembly of the trinuclear complexes, different chiral Co(II)containing species can be present in the reaction mixture, such as the dinuclear complex and some direct adducts between free Co(II) ions and the amino acid. In principle, these chiral Co(II) species can show enhanced VCD signals of their own, ${ }^{14}$ which would make the quantitative analysis more problematic. Conversely, in our case, after the assembly of the dinuclear complexes, the only giant VCD signals stem from Aaa-Co, making the purification steps unnecessary. This renders this particular use of VCD unique and non-comparable with its electronic counterpart.

The assembly dynamics of our system (Scheme 1) was initially monitored by measuring ECD spectra every 30 minutes, in order to evaluate the required reaction time to reach constant values of the ECD ellipticity. We observed that after 1.5 hours the ECD spectrum would remain stable for the following 4 hours. This observation was confirmed with VCD and thereafter, all the VCD measurements were carried out on samples obtained after 2 hours of reaction time.

The VCD spectra are mirror images for the complexes obtained with the enantiomers of each analyte, thus confirming that the overall chirality is induced by the chirality of the amino acid (Fig. 1). In analogy to the already reported trinuclear Co(II) species, ${ }^{17}$ we observe very large VCD signals with the same pattern for different amino acids of the same absolute configuration. For complexes with enantiopure analytes, 20 minutes of acquisition time allowed for a confident quantitation of the dissymmetry factor, $g(\widetilde{v})=\Delta A(\widetilde{v}) / A(\widetilde{v}$ ), even in the cases of the weakest VCD signals (lowest enantiomeric excess, see below). It should be recalled that in any point of the spectrum $g$ is directly proportional to the e.e. 


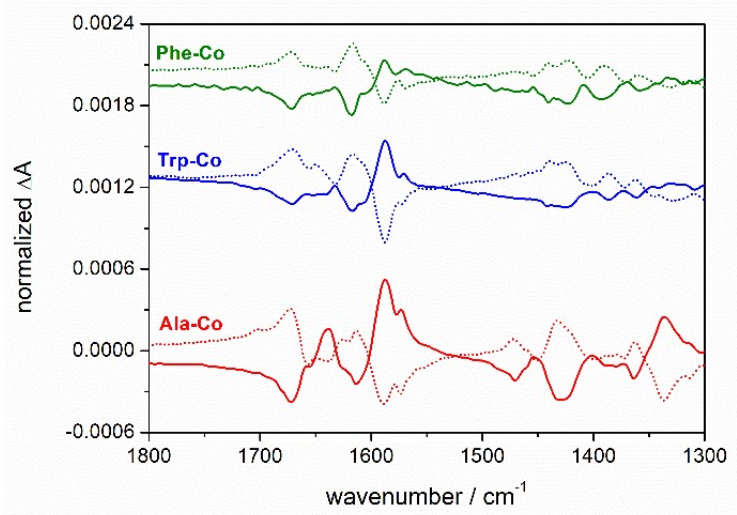

Fig. 1: VCD spectra of probe-analyte complexes for L- (solid lines) and D-enantiomers (dotted lines) of alanine (Ala-Co), tryptophan (Trp-Co) and phenylalanine (Phe-Co) recorded in $\mathrm{CD}_{3} \mathrm{CN}$ solutions. The spectra are normalized to the most intense band of the corresponding IR spectra. In the spectral region centred at $1600 \mathrm{~cm}^{-1}$ we can observe the "AC fingerprint" of these systems: the spectra show a positive (negative) band at $1587 \mathrm{~cm}^{-1}$ and a negative (positive) band at $1617 \mathrm{~cm}^{-1}$ for every L-amino acid (D-amino acid). Spectra are offset for clarity.

As expected, on studying scalemic mixtures, we observed that the intensity of the dichroic bands decreases (in absolute value) when amino acids with lower enantiopurity are employed (Fig. 2), because the oppositely-signed spectra of the two enantiomers subtract, thus translating in lesser dichroic intensities. However, in our case the good signal-to-noise ratio ( $\mathrm{S} / \mathrm{N}$ ) even at $25 \%$ e.e. still allowed for a reliable measurement of the dissymmetry factors (see Fig. S4).

A crucial problem for quantitative applications of VCD spectroscopy is the baseline evolution that occurs during the measurements. The usually long acquisition time necessary to achieve sufficient $\mathrm{S} / \mathrm{N}$ introduces a considerable delay between sample and blank acquisitions, which results in baseline offset and/or slope. The acquisition time for all scalemic mixtures is presently reduced to only $20^{\prime}$, thanks to the enhanced signals.

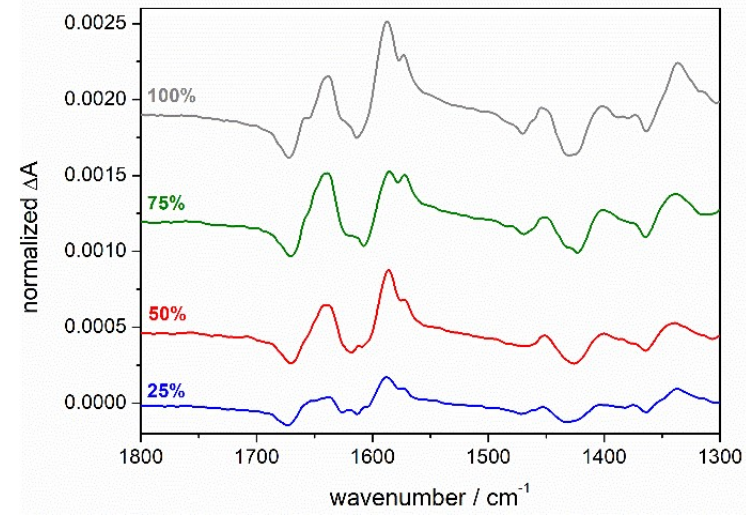

Fig. 2: VCD spectra normalized on the most intense band of the corresponding IR spectra of L-Ala-Co assembled with the amino acid at 100\% (grey), 75\% (green), 50\% (red) and 25\% (blue) of enantiomeric excess. Spectra are shown offset for clarity.

To neutralize any residual baseline drift, we took advantage of two nearby opposite dichroic bands at $1587 \mathrm{~cm}^{-1}$ and $1617 \mathrm{~cm}^{-1}$, using the difference $\Delta g$ between the $g$ values of the positive and negative peaks as the quantitative parameter. In this way, any baseline offset is exactly cancelled, while the effect of the slope is minimal, thanks to the close vicinity of the two lines. In our first investigation, 
we decided to analyse the VCD spectra from the scalemic mixtures of two widely different $\alpha$ aminoacids: Ala-Co and Trp-Co.

The plots of $\Delta g$ vs the enantiomeric excess revealed linear relationships between the enantiomeric compositions of alanine and the intensities of dissymmetry factors of the VCD spectra of Ala-Co (Fig. 3). A linear relationship was observed also in the case of tryptophan (Fig. S6).

The linear dependence between $\Delta g$ of the probe-analyte complexes and the e.e. of the chiral amino acids allows us to build calibration lines, which can be employed to determine the e.e. of a sample with unknown enantiomeric composition.

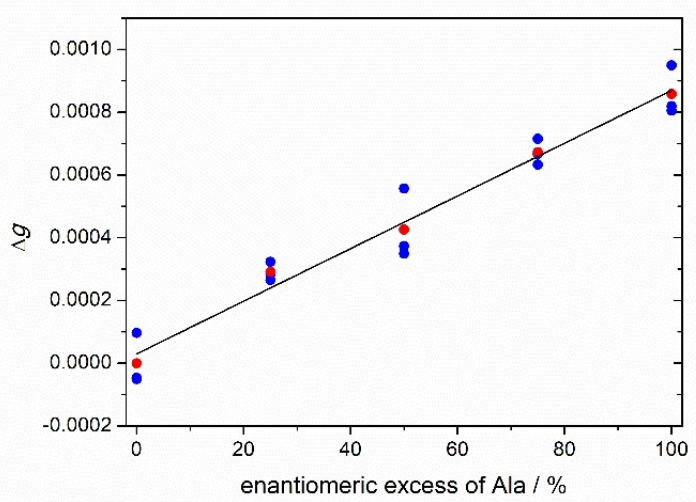

Fig. 3: Plot of the $\Delta \mathrm{g}$ (blue dots), defined as the difference between $\mathrm{g}$ at $1617 \mathrm{~cm}^{-1}$ and $\mathrm{g}$ at $1587 \mathrm{~cm}^{-1}$, recorded for AlaCo vs the enantiomeric excess of alanine. The red dots represent the mean values of the three replicates for each e.e. The linear fit has an R-square value of 0.9868 .

Herein we proposed a proof-of-concept VCD model essay for the determination of the e.e. for a selected class of chiral molecules, exploiting Co(II)-induced VCD signals enhancement for quantitative analysis. In essence, we propose to use a stereodynamic Co(II) complex which should further bind a chiral analyte. As a result of this binding, the enantiomeric forms of the Co-complex become diastereomeric and the Co-complex becomes a chirality reporter. When a scalemic mixture of the analyte is mixed with the Co(II)-complex, then the dichroic signals partly cancel, leaving a VCD intensity, which is directly proportional to the enantiomeric excess. Interestingly, any dependence of the concentration of the analyte or of the reporter is nullified by using a ratiometric metod. Our protocol allowed us to overcome the main problems associated to VCD measurements (weak signals intensities and baseline drift/distortion), even with samples of low enantiomeric excess. The presented method highlights the possibility to further extend the analytical application of VCD to other classes of chiral molecules. In fact, one can take advantage of dynamic covalent chemistry approaches (in our case, imine bond formation and metal coordination) to design new $\mathrm{Co}$ (II) stereodynamic probes, thus developing new protocols in quantitative VCD.

Much remains to investigate in the field of fully understanding the VCD signal enhancement operated by metal ions: the exact role of low-lying electronic transitions centred on the metal and its own paramagnetism, the coupling interaction between metal- (electronic) and ligand-centred (vibrational) transitions, geometrical aspects of this coupling, like its symmetry properties and the distance between the oscillators and the metal centre. Among other groups, we are currently working in this sense. At the same time, we wished to explore the practical consequences of the enhancement, both in qualitative (absolute configuration determination), which we did in our previous report, and presently quantitative (enantiomeric composition) analysis. Hopefully in a near 
future, quantum chemistry calculations will be able to predict the observed effects and provide a further rational approach to the construction of more efficient chirality reporters.

\section{Notes and references}

₹ The ligand (TPA-derivative) and the probe were synthesized according to a reported procedure in acetonitrile. ${ }^{20}$ The probe-analyte complexes were obtained by mixing the amino acid with the desired enantiomeric excess and the probe in methanol in presence of molecular sieves $(4 \AA)$ at room temperature. The solvent was then evaporated and the solid was dissolved in deuterated acetonitrile. VCD spectra were recorded on samples without any purification.

1 L. a Joyce, M. S. Maynor, J. M. Dragna, G. M. Cruz, V. M. Lynch, J. W. Canary and E. V Anslyn, J. Am. Chem. Soc., 2011, 133, 13746-13752.

2 L. You, J. S. Berman and E. V. Anslyn, Nat. Chem., 2011, 3, 943-948.

3 J. Dragna, G. Pescitelli, L. Tran, V. M. Lynch, E. V Anslyn and D. Bari, J. Am. Chem. Soc., 2011, 134, 4398-4407.

4 F. Li, Y. Wang, F. Meng, C. Dai, Y. Cheng and C. Zhu, Macromol. Chem. Phys., 2015, 216, 1925-1929.

$5 \quad$ M. W. Ghosn and C. Wolf, J. Am. Chem. Soc., 2009, 131, 16360-16361.

6 R. Peng, L. Lin, W. Cao, J. Guo, X. Liu and X. Feng, Tetrahedron Lett., 2015, 56, 3882-3885.

7 S. Barman and E. V. Anslyn, Tetrahedron, 2014, 70, 1357-1362.

8 K. M. Spencer, S. J. Cianciosi, J. E. Baldwin, T. B. Freedman and L. A. Nafie, Appl. Spectrosc., 1990, 44, 235-238.

9 C. Guo, R. D. Shah, R. K. Dukor, X. Cao, T. B. Freedman and L. a Nafie, Anal. Chem., 2004, 76, 695666.

10 S. Yamamoto and P. Bouř, Angew. Chemie - Int. Ed., 2012, 51, 11058-11061.

11 C. J. Barnett, A. F. Drake, R. Kuroda, S. F. Mason and S. Savage, Chem. Phys. Lett., 1980, 70, 8-10.

Y. He, X. Cao, L. A. Nafie and T. B. Freedman, J. Am. Chem. Soc., 2001, 123, 11320-11321.

L. A. Nafie, J. Phys. Chem. A, 2004, 108, 7222-7231.

S. R. Domingos, A. Huerta-Viga, L. Baij, S. Amirjalayer, D. A. E. Dunnebier, A. J. C. Walters, M. Finger, L. A. Nafie, B. De Bruin, W. J. Buma and S. Woutersen, J. Am. Chem. Soc., 2014, 136, 3530-3535.

15 S. Lo Piano, S. Di Pietro and L. Di Bari, Chem. Commun., 2012, 11996-11998.

16 S. R. Domingos, F. Hartl, W. J. Buma and S. Woutersen, ChemPhysChem, 2015, 16, 3363-3373.

17 R. Berardozzi, E. Badetti, N. A. Carmo dos Santos, K. Wurst, G. Licini, G. Pescitelli, C. Zonta and L. Di Bari, Chem. Commun., 2016, 52, 8428-8431.

18 T. Kurtán, N. Nesnas, Y. Q. Li, X. Huang, K. Nakanishi and N. Berova, J. Am. Chem. Soc., 2001, 123, 5962-5973.

19 N. Berova, L. Di Bari and G. Pescitelli, Chem. Soc. Rev., 2007, 36, 914-931.

20 F. A. Scaramuzzo, G. Licini and C. Zonta, Chem. - A Eur. J., 2013, 19, 16809-16813.

21 E. Badetti, K. Wurst, G. Licini and C. Zonta, Chem. - A Eur. J., 2016, 22, 6515-6518.

22 J. W. Canary, C. S. Allen, J. M. Castagnetto and Y. Wang, J. Am. Chem. Soc., 1995, 117, 8484-8485.

23 J. M. Castagnetto, X. Xu, N. D. Berova and J. W. Canary, Chirality, 1997, 9, 616-622.

24 J. W. Canary, C. S. Allen, J. M. Castagnetto, Y.-H. Chiu, P. J. Toscano and Y. Wang, Inorg. Chem., 1998, 37, 6255-6262. 